Original Paper http://ajol.info/index.php/ijbcs http://indexmedicus.afro.who.int

\title{
Dynamique spatio-temporelle de l'occupation du sol dans le Moungo, Région du Littoral, Cameroun : influence sur l'expansion des systèmes agroforestiers à base de bananiers
}

\author{
Mélanie Rosine TSEWOUE ${ }^{1,2 *}$, Martin TCHAMBA ${ }^{1,2}$, Marie Louise AVANA ${ }^{2}$, et \\ Armand Delanot TANOUGONG ${ }^{1}$ \\ ${ }^{1}$ Laboratoire de Géomatique Environnemental, Faculté d'Agronomique et des Sciences Agricoles, Université \\ de Dschang, B.P. 222 Dschang, Cameroun. \\ ${ }^{2}$ Département de Foresterie, Faculté d'Agronomique et des Sciences Agricoles, Université de Dschang, B.P. \\ 222 Dschang, Cameroun. \\ *Auteur correspondant ; E-mail : tsewouemel@yahoo.fr; Tél : (+237) $675026986 / 695146389$
}

\section{RESUME}

La présente étude vise à caractériser, sur 32 ans entre 1986 et 2018, les mutations spatio-temporelles qu'a connu la zone du Moungo dans la Région du Littoral au Cameroun en raison de l'expansion des plantations bananières et des diverses pressions dans l'utilisation des ressources. Les images satellitaires Landsat de 1986, 2001 et 2018 ont été exploitées à l'aide de la télédétection et des SIG. La classification non supervisée a permis d'obtenir huit classes d'occupation du sol (forêt dense, forêt claire, plantation et champs de cultures, sols nus, zone habitée, savane herbeuse et surface d'eau). La tendance évolutive des formations végétales est essentiellement régressive pour les forêts dense et claire avec une diminution de 336924,51 ha à 272887,04 ha entre 1986 et 2018, soit un taux de régression de 16,54\% de la superficie totale. Par contre, elle est progressive pour les plantations/champs de cultures, savanes herbeuses, et zones habitées dont les superficies sont passées de 50 231,24 ha en 1986 à 111 325,41 ha en 2018 soit un taux de progression de 15,77\%. Les facteurs de cette dégradation sont principalement d'ordres humains (agriculture, surpâturage et démographie galopante) et se traduit par la fragmentation des formations végétales naturelles au profit des surfaces cultivées et des zones d'habitations.

(C) 2020 International Formulae Group. All rights reserved.

Mots clés : Classes d'occupation du sol, facteurs de dégradation, Systèmes d'Information Géographique, Moungo-Cameroun.

\section{Spatio-temporal dynamic of land use change in the Moungo division, Littoral Region, Cameroon : influence on the expansion of banana-based agroforestry systems}

\begin{abstract}
This study aims to characterize, over a 32-year period between 1986 and 2018, the spatial and temporal changes that occurs in the Moungo division located in the Littoral Region of Cameroon has undergone due to expansion bananas plantation and various pressures in the natural resources use. Landsat satellite images from
\end{abstract}


1986, 2001 and 2018 were exploited using remote sensing and GIS. The unsupervised classification yielded eight land use classes (dense forest, open forest, plantation and croplands, bare soil, inhabited area, herbaceous savannah and water plans). The evolutionary trend of vegetation formations is essentially regressive for dense and open forest with a decrease from 336924.51 ha to 272887.04 ha between 1986 and 2018, indicating a regression rate of $16.54 \%$ of the total area. On the other hand, it is progressive for plantations/croplands, herbaceous savannas, and inhabited areas, whose areas increased from 50231.24 ha in 1986 to 111325.41 ha in 2018 , thus an increment rate of $15.77 \%$. The degradation factors are mainly anthropogenic and include agriculture, overgrazing and increasing human population. As a result, the natural vegetation is being fragmented and transformed into cultivated areas and human inhabitations.

(C) 2020 International Formulae Group. All rights reserved.

Keywords : Land use classes, degradation factors, Geographical Information Systems, Moungo-Cameroon.

\section{INTRODUCTION}

Entre 1900 et 2000, la FAO a estimé que le continent africain a perdu 52 millions d'hectares de forêts, représentant $56 \%$ de la réduction du couvert forestier mondial (FAO, 2014). Cette dégradation des ressources naturelles est principalement imputable aux pratiques de gestion non durables (déboisement, surpâturage, forte démographie toujours croissante, besoin énergétique croissant, surexploitation des ressources fourragères, exploitation industrielle accrue) (Abdourhamane et al., 2012; Ballo et al., 2016 ; Temgoua et al., 2018a). Le rythme de dégradation de ces forêts et les menaces d'extinction écologiques des ressources dont elles regorgent en sont les préoccupations en matière de gestion durable de l'environnement. Dans le bassin du Congo, le taux de déforestation a connu une nette progression entre les périodes 1990 - 2000 et entre 20002005 , respectivement de $0,09 \%$ à $0,17 \%$ (Bozongo, 2018).

$\mathrm{Au}$ Cameroun, on note un recul de la surface forestière d'environ 220000 ha par an (FAO, 2010). D'après Ciza et al. (2012), les forêts denses représentaient $71 \%$ du couvert forestier, soit environ 1909104 ha, contre 29\% des forêts non denses, soit environ 7763 960 ha. Ndobe et Mantzel (2014), estiment qu'entre 1990 et 2010, les surfaces boisées du pays ont régressé d'environ 4400000 ha, soit $18,1 \%$ du couvert forestier. Les principaux facteurs de cette déforestation au Cameroun sont la conversion des forêts pour l'agriculture (qui représente $80 \%$ de la perte du couvert forestier), les besoins de plus en plus croissants des populations en bois de chauffage et l'exploitation forestière illégale (Tanougong, 2019). Le Moungo, dans le Littoral, ne fait pas exception à cette réalité. Cette zone, autrefois, regorgeait une biodiversité très importante au sein desquels on dénombrait plusieurs formes d'utilisations dont vivent les populations de cette localité. Cette région fait, de nos jours, l'objet d'une menace exacerbée de ses ressources à cause de la forte pression humaine sur les terres, ce qui met en péril les espaces naturels. En même temps, les pratiques agricoles s'intensifient de plus en plus à travers la colonisation des nouvelles terres pour la mise en place des cultures intensives des bananes, d'ananas et de fruitiers. Face à cette situation, il devient important de mener des études spécifiques qui permettront d'avoir des bases soutenables pour envisager une gestion durable des ressources de cette localité. La télédétection et la cartographie offrent une immense source de données pour étudier la dynamique spatiale et temporelle des paramètres environnementaux. Elles peuvent fournir des informations synoptiques en temps opportun pour l'identification et la surveillance des territoires locaux (Smith, 2012). De plus, elles jouent un rôle capital dans les applications telles que l'évaluation des dommages environnementaux, le suivi de l'occupation du sol, la planification urbaine, l'évaluation des sols et des rendements des cultures (Avakoudjo et al., 2014). Cette étude a pour objectif 
d'apprécier les changements de l'occupation des terres dans le Moungo à partir de données cartographiques de la localité. Elle se fonde sur l'hypothèse que l'expansion des plantations agro-industrielles surtout des bananiers dans la zone combinée aux effets des pratiques de gestion non durables interviennent dans la dynamique spatio-temporelle de la zone du Moungo.

\section{MATERIEL ET METHODES}

\section{Situation géographique du Moungo}

Le Département du Moungo dont le chef-lieu est Nkongsamba, est situé dans la partie Ouest du Cameroun oriental. Le fleuve, qui lui a donné son nom, le sépare du Cameroun occidental sur tout son cours inférieur. Le Département s'étend du sud au nord sur près de $140 \mathrm{~km}$, mais sa largeur ne dépasse pas $40 \mathrm{~km}$ et couvre une superficie de 372300 ha. Il est compris entre les latitudes 4³’20 Nord et les longitudes 9॰57’30 Est (Figure 1).

Le relief du Moungo est généralement accidenté, constitué de collines, de plateaux, de bas-fonds et de vallées. Ce département est entouré de massifs montagneux dont les plus importants sont : le Manengouba à $2400 \mathrm{~m}$, Koupé à $2070 \mathrm{~m}$ et, Nlonako à $1800 \mathrm{~m}$. Le climat est de type Equato-guinéen, la pluviométrie est toujours élevée (plus de 2000 $\mathrm{mm}$ ) mais présente d'importante variations locales. La répartition de la pluviométrie a une allure particulière. Un seul maximum en septembre pour une pluviométrie étalée sur dix mois. La saison des pluies s'établit progressivement à partir du mois de mars et après un pallier en juin ; entre juillet et octobre, on observe de très fortes pluies. La saison sèche ne dure pratiquement que deux mois. Les sols sont d'origine volcaniques (andosols) et très fertiles. On y retrouve des sols bruns eutrophes formés sur des coulées récentes de basalte bulbeux, les sols ferrallitiques typiques dont certains sont intensément cultivés depuis de nombreuses années, et qui présentent des signes de dégradation et nécessitent par ailleurs un travail sérieux de remises en état.

La végétation du Moungo se présente sous l'aspect d'une forêt dégradée. Toutefois, on y retrouve des forêts secondaires et des galeries forestières. A certains endroits, elle est fortement constituée d'agroforesterie et de savane. Les principales espèces florales qu'on y retrouve sont Guibourtia demeusei, Milicia excelsa, Lophira alata, Ceiba pentandra, Pterocarpus mildbraedii, Erythroxylum mannii, Distemonanthus benthamianus, ... Ces forêts regorgent des produits forestiers non ligneux (PFNLs) tels que le Ricinodendron heudelotii, Dacryodes macrophylla, Garcinia kola, Bambusa sp et Raphia sp, ainsi que diverses plantes médicinales. La disparition de la forêt dans certaines localités est une conséquence de la coupe anarchique d'arbres. Le Département du Moungo est une grande zone agricole de la Région du Littoral. En plus de l'agriculture de subsistance pratiquée par les populations, plusieurs plantations agroindustrielles y sont implantées et cultivent entre autres la banane dessert, les ananas, les fleurs, le palmier à huile, le poivre et la papaye solo. Par ailleurs, les cultures de rentes telles que le café, le cacao, la culture des fruits (citron, mangues, oranges, safou, etc.), la culture des tubercules et des légumes appartiennent aux paysans.

Le troisième Recensement Général de la Population et de 1'Habitat ( $3^{\mathrm{e}} \mathrm{RGPH}$ ), réalisé en 2005, créditait le Département du Moungo de 379241 habitants soit un taux d'urbanisation de $84,7 \%$ et une densité de 101,9 habitants au $\mathrm{km}^{2}$. Selon l'agence régionale de l'Institut National de la Statistique du Littoral, cette population serait de 506857 habitants avec une densité de 136,1 habitants au $\mathrm{km}^{2}$. (ASRT, 2015). Cette population est essentiellement rurale avec comme activité principale l'agriculture et le commerce.

\section{Méthodologie}

La démarche méthodologique adoptée est basée sur une approche cartographique, 
analytique et diachronique basée sur l'utilisation de la télédétection et des systèmes d'information géographique. Trois images satellitaires Landsat TM de 1986 et ETM+ et OLI-TIRS de 2001 et de 2018 ont été utilisées. La résolution de chaque image est de $30 \mathrm{~m}$. La projection adoptée est WGS 1984, UTM-Zone 33 N. L'image Digital Globe de résolution 2,5 $\mathrm{m}$ a été utilisée pour la finalisation de la carte d'occupation des sols. L'image ALOS PALSAR de 2006 de résolution 12,5 m a été utilisée pour la réalisation du modèle numérique de terrain (MNT) (Tableau 1). La validation finale des différentes cartes d'occupations des sols, s'est faite à l'aide de matrice de confusion des pixels et des données issues des visites de terrain où un récepteur de poche GPS (Global Positionning System) de marque GARMIN a été utilisée pour localiser la position des différents points de contrôle. Un appareil photographique a été utilisé pour filmer les sites importants (Forêts primaire, secondaire, champs de culture, plantations industrielle, savane).

Les superficies des différentes classes d'occupation du sol ont été calculées au moyen du logiciel Arc Gis 10.1. Ce qui a permis d'effectuer l'étude diachronique des situations des années de référence afin de faire ressortir la dynamique du couvert végétal de la zone d'étude. La classification d'image s'est portée sur la classification supervisée et a consisté à définir la nomenclature des différents types d'occupations des sols basée sur le model LCCS (Land Cover Classification System) (Hussain et al., 2013). La classification dirigée par la méthode de maximum de vraisemblance a été appliquée pour chacune des images de $1986 ; 2001$ et 2018 et a permis l'identification des différentes classes d'occupation des terres. Les images satellitaires ont subi divers traitements : après le mosaïquage des différentes bandes, il a été effectué dans un premier temps un rehaussement de la qualité des images, c'est-à-dire l'amélioration de leurs contrastes et la composition colorée. Ensuite, elles ont subi des corrections géométriques qui ont consisté à recaler l'image sur un référentiel géographique. La zone d'intérêt a été extraite à partir des limites de la zone d'étude.

Des missions de terrain «vérité terrain » ont été organisées par la suite pour la validation des limites des différentes unités d'occupation des terres issues de l'interprétation ainsi que la précision de celleci par la confrontation des résultats cartographiques à la réalité du terrain. Cette étape du traitement a permis aussi de vérifier les sites incertains et rectifier les erreurs d'interprétation, afin de valider les travaux cartographiques menés.

\section{Traitement des données}

La dynamique spatiale est basée sur l'appréciation de l'évolution des états et la comparaison de la superficie des différentes unités d'occupations du sol. Pour l'analyse statistique de la dynamique d'occupation, le taux de stabilité, de régression ou de progression, des unités paysagiques est calculé d'une année à l'autre. Ce calcul a été fait à l'aide de la formule appliquée par Inoussa et al. (2011), pour mesurer la croissance des agrégats macroéconomiques entre deux périodes données.

La variation considérée ici est la superficie (S). Ainsi, pour S1 et S2, correspondant respectivement à la superficie d'une catégorie d'occupation des sols en différentes périodes, les taux de variation des superficies ont été calculés par la formule

$\mathbf{T v}(\%)=\left[\frac{\mathrm{S} 2}{\mathrm{~S} 1}-\mathbf{1}\right] * \mathbf{1 0 0}$

- $\mathrm{S} 2-\mathrm{S} 1=$ négatif, on conclut une régression du couvert végétal de l'année 1 à 2.

- $\mathrm{S} 2$-S1 = positif, on parle d'une augmentation du couvert végétal de l'année 1 à 2 .

- $\quad \mathrm{S} 2-\mathrm{S} 1=$ nul, on parle de stabilité du couvert végétal de l'année 1 à 2 . 


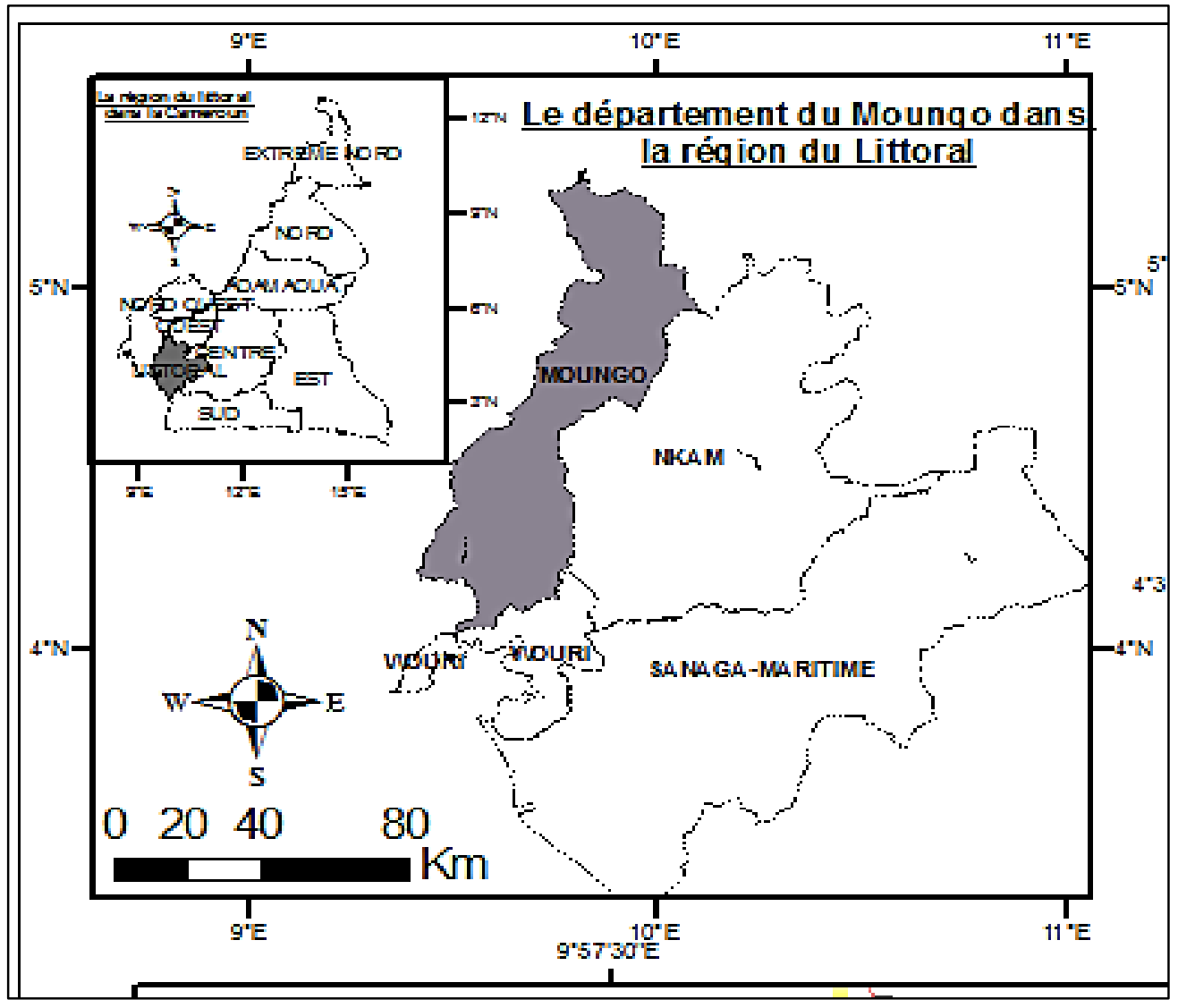

Figure 1 : Situation de la zone d'étude.

Tableau 1 : Ensemble des scènes d'images utilisées pour la cartographie de l'occupation des sols du Moungo.

\begin{tabular}{|c|c|c|c|c|c|}
\hline Capteurs & Année & Nombre de bandes & Résolution (m) & Sources & Path/Row \\
\hline Landsat TM & $04 / 12 / 1986$ & 9 & 30 & glovis.usgs.gov & $184 / 057$ \\
\hline $\begin{array}{ll}\text { Landsat } & 7 \\
\text { ETM+ }\end{array}$ & $05 / 12 / 2001$ & 9 & 30 & glovis.usgs.gov & $184 / 057$ \\
\hline $\begin{array}{ll}\text { Landsat } & 7 \\
\text { ETM+ }\end{array}$ & $17 / 12 / 2010$ & 9 & 30 & glovis.usgs.gov & $184 / 057$ \\
\hline $\begin{array}{l}\text { Landsat } 8 \text { OLI- } \\
\text { TIR }\end{array}$ & $07 / 12 / 2015$ & 14 & 30 & glovis.usgs.gov & $184 / 057$ \\
\hline $\begin{array}{l}\text { Landsat } 8 \text { OLI- } \\
\text { TIR }\end{array}$ & $15 / 12 / 2018$ & 14 & 30 & glovis.usgs.gov & $184 / 057$ \\
\hline $\begin{array}{l}\text { ALOS } \\
\text { PALSAR }\end{array}$ & // & 2 & 12,5 & $\begin{array}{l}\text { Asf.alaska.edu/s } \\
\text { ar-data }\end{array}$ & $104 / 42$ \\
\hline Digital Globe & $23 / 12 / 2015$ & 3 & 2,5 & Google earth & // \\
\hline
\end{tabular}




\section{RESULTATS}

\section{Dynamique \\ spatio-temporelle de \\ l'occupation du sol dans le Moungo entre 1986 et 2018}

Cette dynamique spatio-temporelle concerne l'état de l'occupation du sol, l'étude de la dynamique de l'occupation du sol et les facteurs de cette dynamique.

\section{Etat de l'occupation du sol en 1986}

En 1986, d'après la répartition de l'occupation du sol dans le Moungo, le paysage était très boisé, occupé en grande partie par les forêts denses $(69,57 \%)$ équivalant à une superficie de 269402,94 ha et partiellement par les forêts claires $(17,44 \%)$ soit 67521,61 ha. Les plantations et les champs de cultures couvraient environ une superficie de 37205,44 ha soit $9,61 \%$ du paysage et sont en majorité présentes le long des routes et autour des zones habitées qui représentaient environ $1,08 \%$ de la superficie totale. La Figure 2 permet de caractériser l'évolution de l'état d'occupation du sol en 1986.

\section{Etat de l'occupation du sol en 2001}

Jusqu'en 2001, le paysage est resté très boisé avec une dominance des forêts denses qui s'étendaient sur une superficie d'environ 266939,36 ha soit $68,93 \%$ de la superficie totale du Moungo. Les forêts claires s'étendaient sur 65474,64 ha soit $16,91 \%$ de la superficie totale. Les plantations et les champs de cultures ont subi une baisse de superficie (20059,47 ha) soit 5,17\% de superficie. Les zones habitées ont augmenté et occupaient déjà une superficie de 4276 ha soit $1,10 \%$ de la superficie totale du Département. Les savanes herbeuses, par contre, ont occupé une superficie de 28109,43 soit $7,26 \%$ de la superficie totale du Moungo (Figure 3). Elles sont de plus en plus très visibles dans les plantations et les champs de culture abandonnées. Cette figure montre également l'évolution de l'état d'occupation du sol du Moungo au cours des années 2001.

\section{Etat de l'occupation du sol en 2018}

Les images satellitaires prises en 2018 (Figure 4) du Département du Moungo révèlent une importante variation du couvert végétal comparativement aux images prises en 1986 et en 2001. Il ressort de cette figure que le paysage est de moins en moins boisé. Nous avons observé en augmentation considérable des plantations industrielles et des champs de cultures, avec une métamorphose du paysage agricole (due au remplacement des caféiers par des bananiers et bananiers plantains et les fruitiers tels que les papayers). La savane herbeuse occupe déjà une superficie de 57654,63 ha soit $14,89 \%$ de la superficie totale. De même, les plantations et les champs de cultures occupent une superficie de 39364,56 ha soit $10,16 \%$ de la superficie totale. Par contre, la superficie de la forêt dense est passée de 269402,94 à 205399,98 ha soit une baisse de $53,04 \%$ de la superficie totale.

\section{Dynamique de l'occupation du sol De 1986 à 2001}

Les différentes formations végétales ont subi des modifications sensibles pour certaines entre 1986 et 2001 (Tableau 2). Les forêts denses et claires ont vu leur superficie diminuer respectivement de 2463,5 et de 2046,97 ha soit une régression de 0,64 et $0,53 \%$. De même, les plantations et les champs de cultures ont aussi régressé de 17145,97 ha soit une perte de $4,43 \%$. Par contre, les zones habitées et les savanes herbeuses ont vu leur superficie s'accroître. La superficie des savanes herbeuses a augmenté de 22021,21 ha soit une augmentation de 5,68\%. Celle des zones habitées a subi une légère augmentation de 81,79 ha, soit une augmentation de $0,02 \%$.

Les résultats ont montré que les plantations et les champs de cultures ont été abandonnés de plus de 17145,97 ha. Ce résultat traduit une baisse de superficie cultivable probablement due à l'abandon des plantations qui étaient en majorité caféières à cause des multiples crises comme la chute drastique des prix du café et l'arrêt des subventions des agriculteurs par l'Etat. Bon nombre de plantations restées sont des bananeraies surtout du Centre Africain de Recherche en Bananiers et Plantains (CARBAP) et des Plantations du Haut Penja (PHP), Cameroon Development Corporation (CDC).

\section{De 2001 à 2018}

L'évolution des superficies d'unités d'occupations du sol entre 2001 et 2018 est présentée dans le Tableau 3. La tendance observée montre qu'en dehors des forêts denses qui continuent de régresser, toutes les 
autres unités d'occupations du sol ont progressé. Les forêts denses ont connu une régression de 61539,4 ha soit $15,89 \%$ de la superficie totale. Ces forêts sont détruites aux profits des autres formations végétales telles que les plantations industrielles et les champs de cultures, les savanes herbeuses et les sols nus. Les forêts claires ont progressé de 2012,4 ha soit $0,52 \%$. Les plantations et les champs de cultures, contrairement à la période de 1986 à 2001, ont subi une progression de superficie de 19305,09 ha soit $5,99 \%$. Cette phase traduit la phase de la relance des activités agricoles après la période de crise. De même, on constate que les sols nus, la zone habitée, les savanes herbeuses et les surfaces d'eau ont tous progressé respectivement de $1,53 \%, 0,44 \%$, $7,63 \%$ et $0,78 \%$.

\section{De 1986 à 2018}

Le Tableau 4 présente l'évolution des unités d'occupations du sol sur 32 ans dans le Moungo. Entre 1986 et 2018 on observe une forte régression des forêts denses de plus de 64002,9 ha soit $16,53 \%$ de la superficie totale du Département du Moungo au profit des savanes herbeuses qui ont progressé de 13,31\% soit une superficie de 51566,41 ha. Les forêts claires également ont subi une régression de $0,01 \%$. Par contre, les plantations et les champs de culture, les sols nus, les zones habitées, et la surface d'eau ont subi une progression respectivement de $0,56 \%, 1,44 \%, 0,46 \%$ et, $0,76 \%$.

\section{Synthèse de l'occupation des sols du Moungo en 1986, 2001 et 2018}

La Figure 5 montre une représentation graphique de la tendance de l'évolution des changements d'occupation du sol du Moungo pour les 3 périodes. Nous pouvons constater que les changements les plus remarquables ont eu lieux entre 2001 et 2018. Les années 1986 et 2001, sont les périodes qui semblent n'avoir pas beaucoup évolué avec une variation plus ou moins constante des superficies. On observe aussi que les forêts denses dans les 3 périodes ont beaucoup régressé, indiquant une perte importante du couvert forestier dans la localité. Par contre, les savanes herbeuses ont subi une importante progression. Les surfaces qui sont restées relativement stables dans les trois périodes sont les forêts claires, les surfaces d'eau, et les zones habitées. En effet, les forêts denses sont passées de 269402,9 ha en 1986, à 266939,4 ha en 2001 et à 205400 ha en 2018. Les plantations et les champs de cultures ont régressé de 1986 à 2001 (de 37205,44 à 20059,47ha), puis ont subi une forte progression de 2001 à 2018 (de 20059,47 ha à 39364,56 ha) (Figure 6).

Ces résultats montrent également que l'augmentation des espaces de cultures contribue à la diminution des formations végétales naturelles. De même, l'accroissement de la population constitue aussi un facteur de destruction du couvert végétal car une forte croissance démographique a pour conséquence la forte demande en ressources alimentaires, énergétiques et en espaces d'habitation. En effet, la zone habitée est passée de 3194,21 en 1986, à 4276 en 2001 et à 5988,96 en 2018.

\section{Vecteurs de changement dans l'occupation des terres dans le Moungo}

La dynamique d'occupation des terres du Moungo est causée par de nombreux facteurs afférents aux activités anthropiques. L'augmentation de la population du Moungo entre 1987 et 2014 constitue la première cause de la dégradation des autres formations végétales de la localité. Entre 1987 et 2001, la population a augmenté très peu (Figure 7). Entre 2001 et 2005 on observe une augmentation de la population de 379241 à 452722 habitants. Entre 2005 et 2014, on note une très forte augmentation de la population, de 452722 habitants à 776517 habitants.

La pression des populations sur le couvert végétal entraine la diminution du peuplement forestier au profit des espaces agricoles. Cette disparition des formations végétales entraînera l'augmentation des gaz à effet de serre dans l'atmosphère, interrompant ainsi l'équilibre écologique avec pour conséquence: la récession pluviométrique, l'augmentation de la température, l'érosion hydrique qui favorisera la dégradation des éléments fertilisants du sol, la baisse des rendements sur les sols dégradés. Les espèces ligneuses épargnées de la destruction par les agriculteurs dispersées dans les champs et autour des agglomérations sont celles à valeur culturelle et économique comme notamment Dacryodes edulis, Persea americana, Elaeis guineensis, Garcinia kola et Mangifera indica. 
M. R. TSEWOUE et al. / Int. J. Biol. Chem. Sci. 14(2): 486-500, 2020

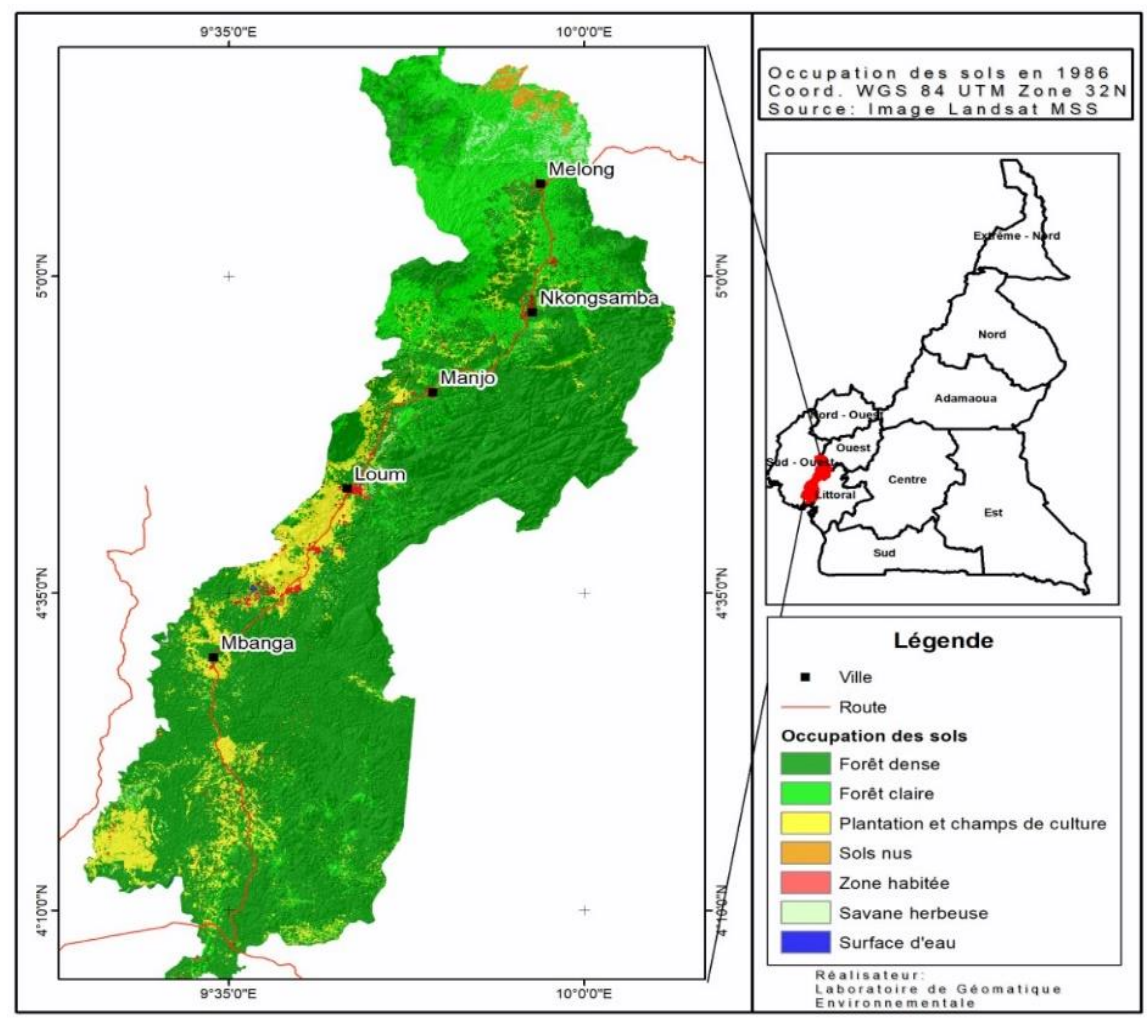

Figure 2 : Occupation du sol dans la zone du Moungo en 1986.

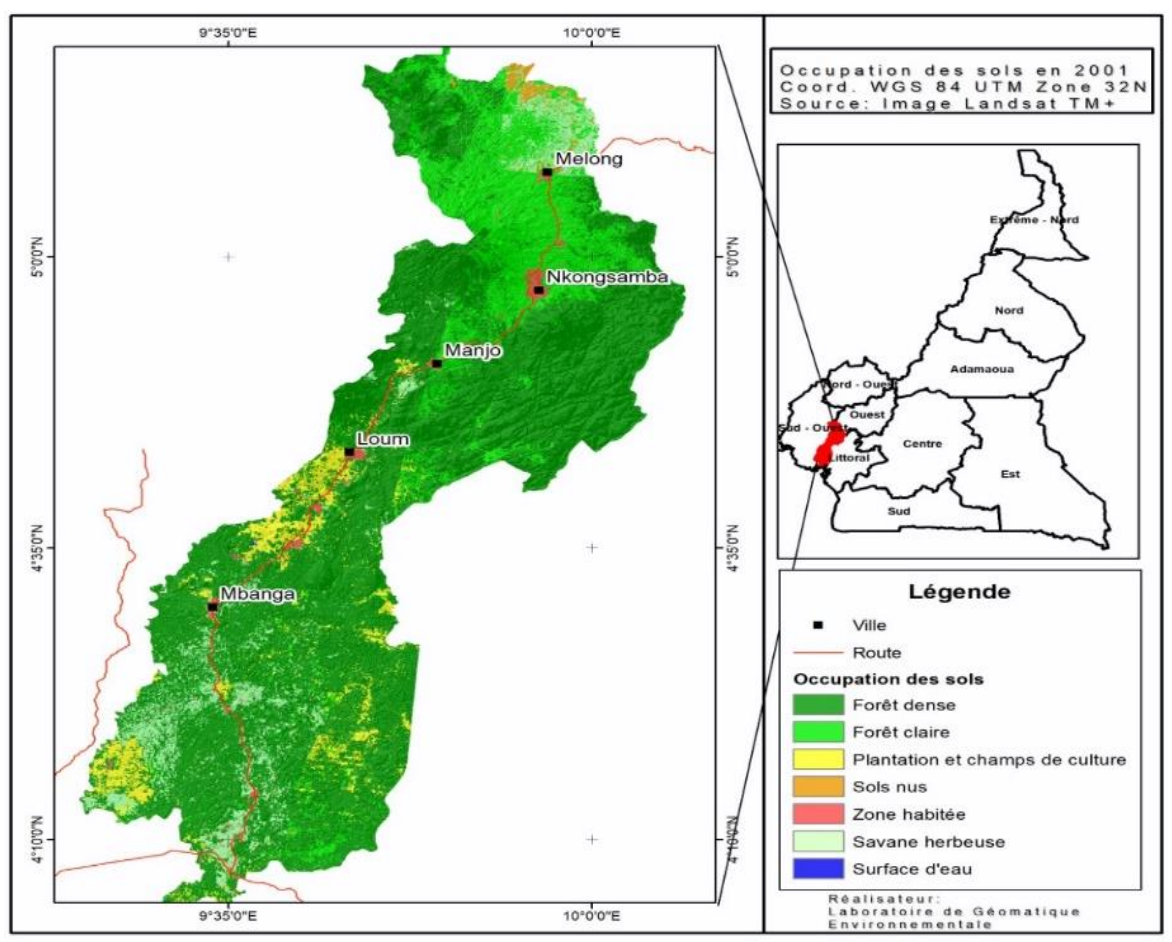

Figure 3 : Occupation du sol dans la zone du Moungo en 2001. 


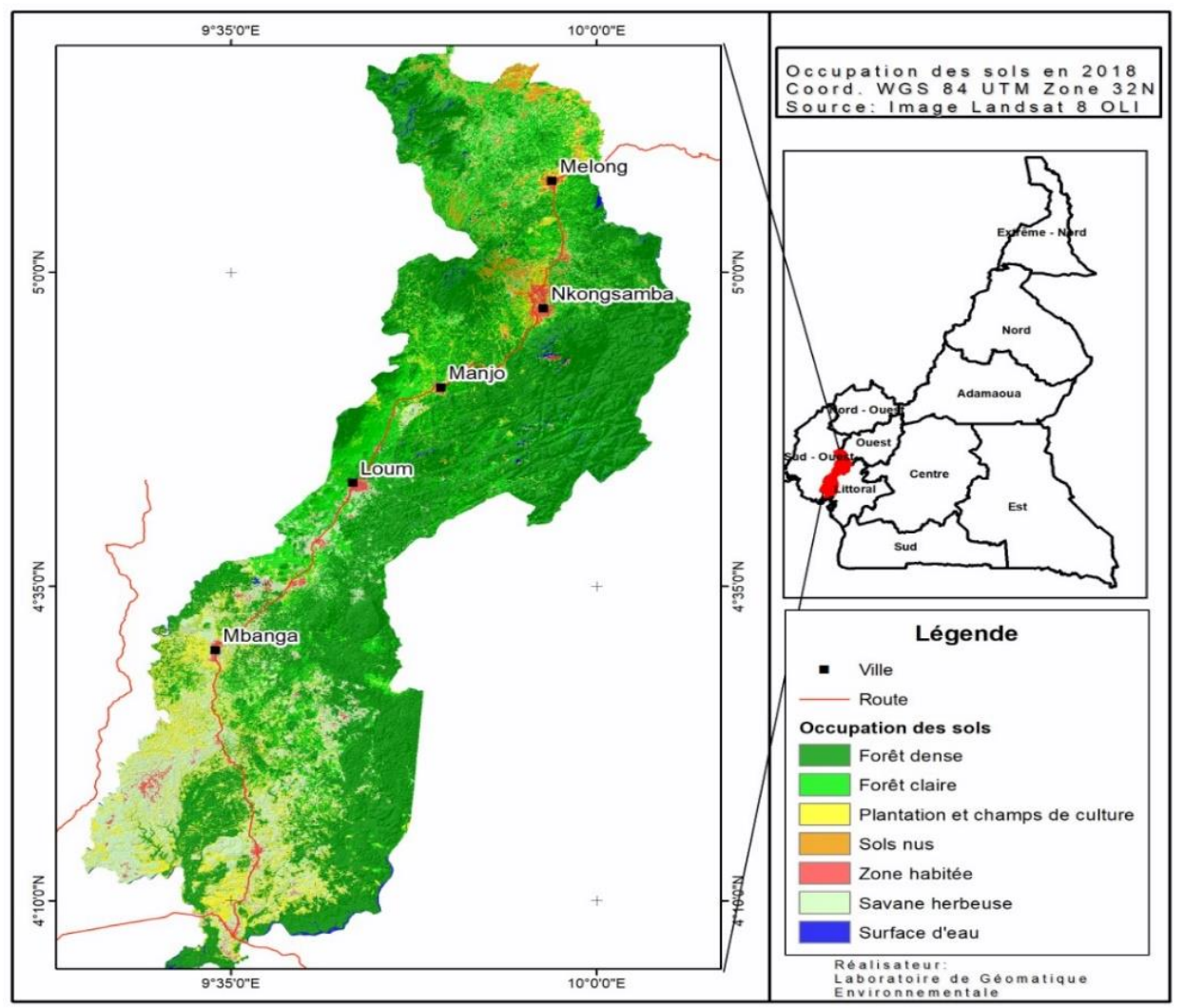

Figure 4 : Occupation du sol dans la zone du Moungo en 2018.

Tableau 2 : Variation des superficies d'occupations de sol de 1986 à 2001.

\begin{tabular}{|c|c|c|c|c|}
\hline \multirow[b]{2}{*}{ Unité d'occupation des sols } & \multicolumn{2}{|c|}{ Superficie (ha) } & \multicolumn{2}{|c|}{ Variation des superficies } \\
\hline & 1986 & 2001 & (ha) & $\%$ \\
\hline Forêt dense & 269402,9 & 266939,4 & $-2463,5$ & $-0,64$ \\
\hline Forêt claire & 67521,61 & 65474,64 & $-2046,97$ & $-0,53$ \\
\hline $\begin{array}{l}\text { Plantation et champs de } \\
\text { culture }\end{array}$ & 37205,44 & 20059,47 & $-17145,97$ & $-4,43$ \\
\hline Sols nus & 2743,37 & 2370,6 & $-372,77$ & $-0,1$ \\
\hline Zone habitée & 4194,21 & 4276 & $+81,79$ & $+0,02$ \\
\hline Savane herbeuse & 6088,22 & 28109,43 & $+22021,21$ & $+5,68$ \\
\hline Surface d'eau & 106,6484 & 32,94 & $-73,71$ & $-0,02$ \\
\hline Total & 387262,4 & 387262,4 & & \\
\hline
\end{tabular}


Tableau 3 : Variation des superficies d'occupations de sol de 2001 à 2018.

\begin{tabular}{lllll}
\hline & \multicolumn{2}{l}{ Superficie (ha) } & \multicolumn{2}{l}{ Variation des superficies } \\
\cline { 2 - 6 } Unité d'occupation des sols & $\mathbf{2 0 0 1}$ & $\mathbf{2 0 1 8}$ & (ha) & \% \\
\hline Forêt dense & 266939,4 & 205400 & $-61539,4$ & $-15,89$ \\
Forêt claire & 65474,64 & 67487,04 & $+2012,4$ & $+0,52$ \\
$\begin{array}{lllll}\text { Plantation } \\
\text { culture }\end{array}$ & & 39364,56 & $+19305,09$ & $+4,99$ \\
Sols nus & & & & \\
Zone habitée & 20059,47 & & & \\
Savane herbeuse & 2370,6 & 8317,26 & $+5946,66$ & $+1,53$ \\
Surface d'eau & 4276 & 5988,96 & $+1712,96$ & $+0,44$ \\
Total & 28109,43 & 57654,63 & $+29545,2$ & $+7,63$ \\
\hline
\end{tabular}

(-) régression, (+) progression

Tableau 4 : Variation des superficies d'occupations de sol de 1986 à 2018.

\begin{tabular}{lllll}
\hline \multirow{2}{*}{$\begin{array}{l}\text { Unité } \\
\text { des sols }\end{array}$} & \multicolumn{2}{l}{ Superficie (ha) } & \multicolumn{2}{l}{ Variation des superficies } \\
\cline { 2 - 5 } & & $\mathbf{2 0 1 8}$ & (ha) & \% \\
\hline Forêt dense & 269402,9 & 205400 & $-64002,9$ & $-16,53$ \\
Forêt claire & 67521,61 & 67487,04 & $-34,57$ & $-0,01$ \\
Plantation et champs de & & 39364,56 & $+2159,12$ & $+0,56$ \\
culture & 37205,44 & & & \\
Sols nus & 2743,37 & 8317,26 & $+5573,89$ & $+1,44$ \\
Zone habitée & 4194,21 & 5988,96 & $+1794,75$ & $+0,46$ \\
Savane herbeuse & 6088,22 & 57654,63 & $+51566,41$ & $+13,31$ \\
Surface d'eau & 106,6484 & 3050,01 & $+2943,36$ & $+0,76$ \\
Total & $\mathbf{3 8 7 2 6 2 , 4}$ & $\mathbf{3 8 7 2 6 2 , 4}$ & & \\
\hline
\end{tabular}

(-) régression, (+) progression

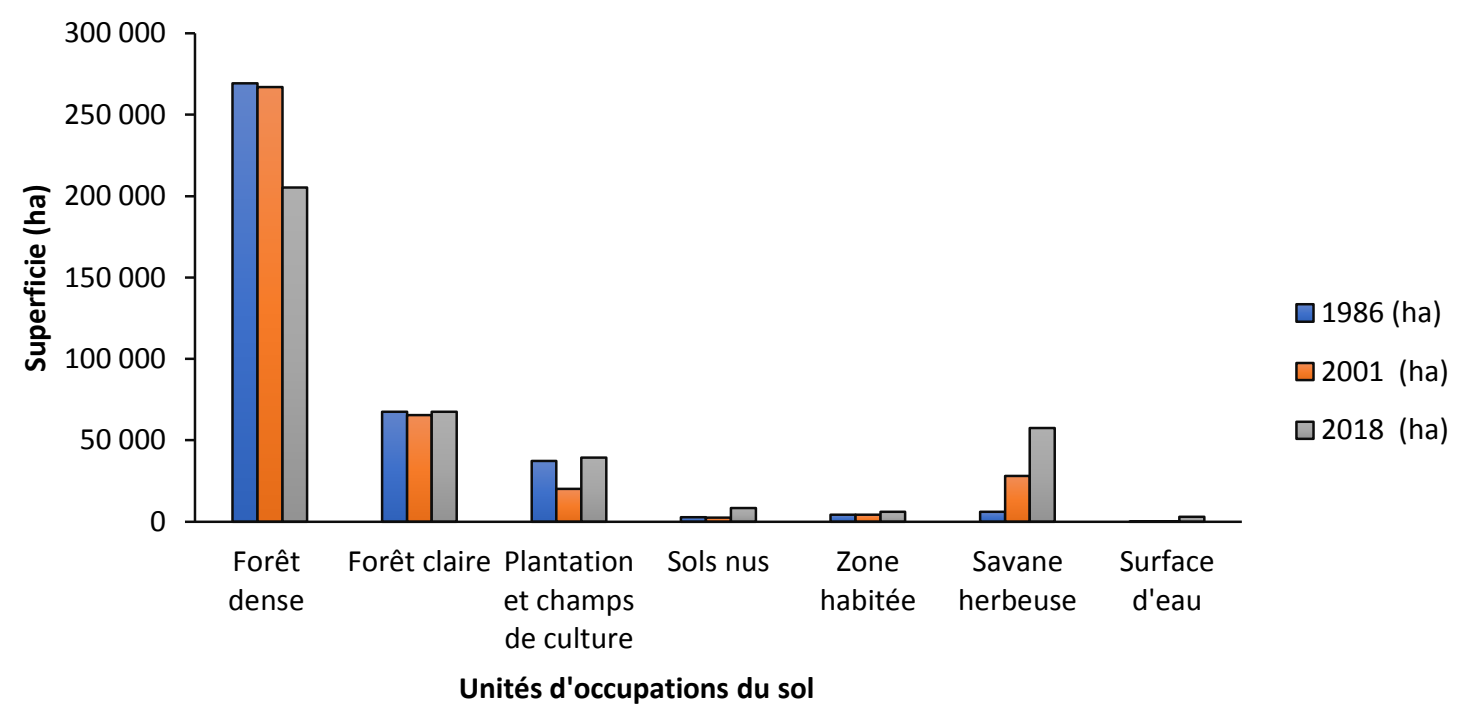

Figure 5 : Synthèse de la distribution de l'occupation des sols entre 1986, 2001 et 2018. 

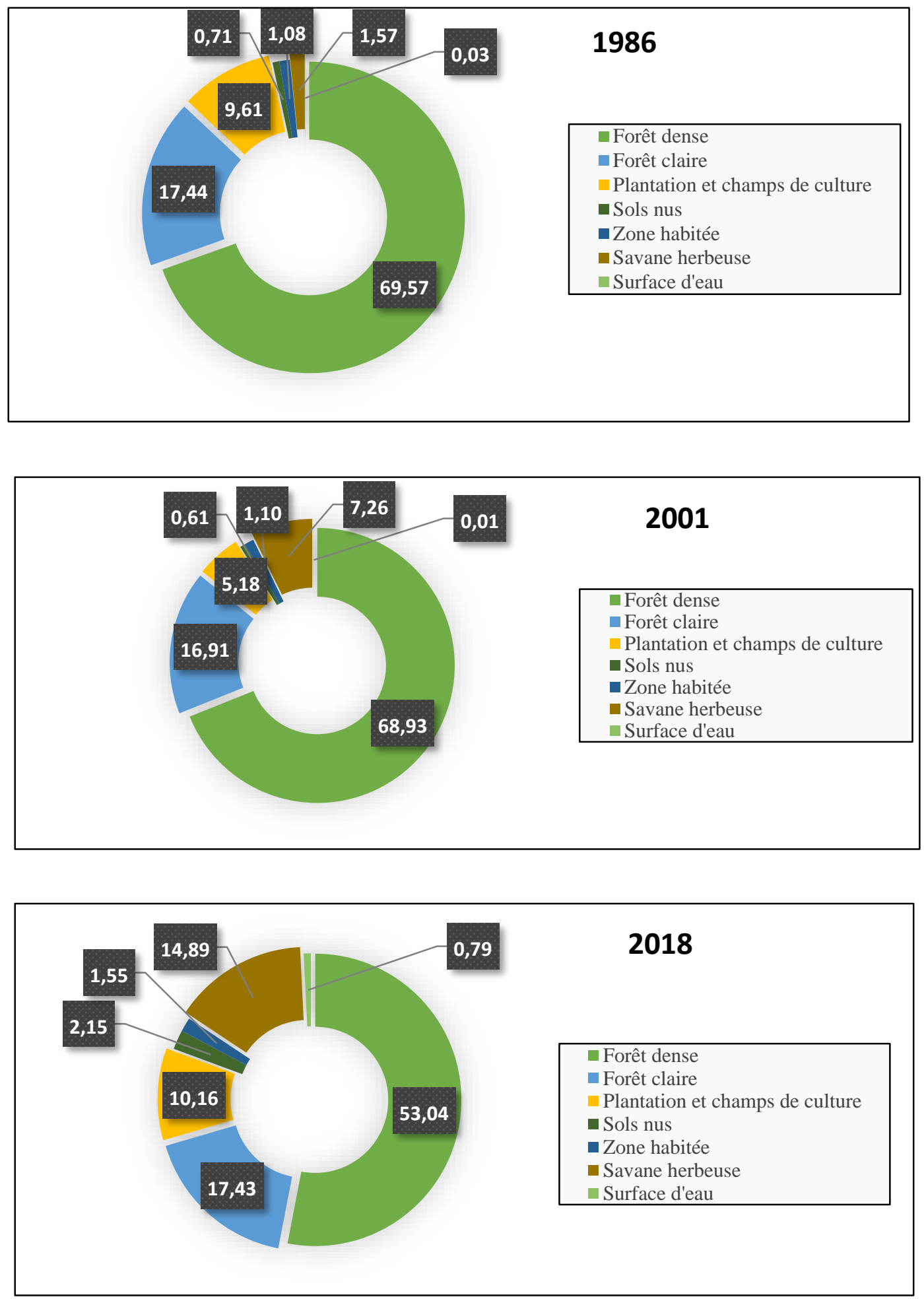

Figure 6 : Synthèse de l'occupation du sol entre 1986, 2001 et 2018 suivant les pourcentages dans le Moungo. 


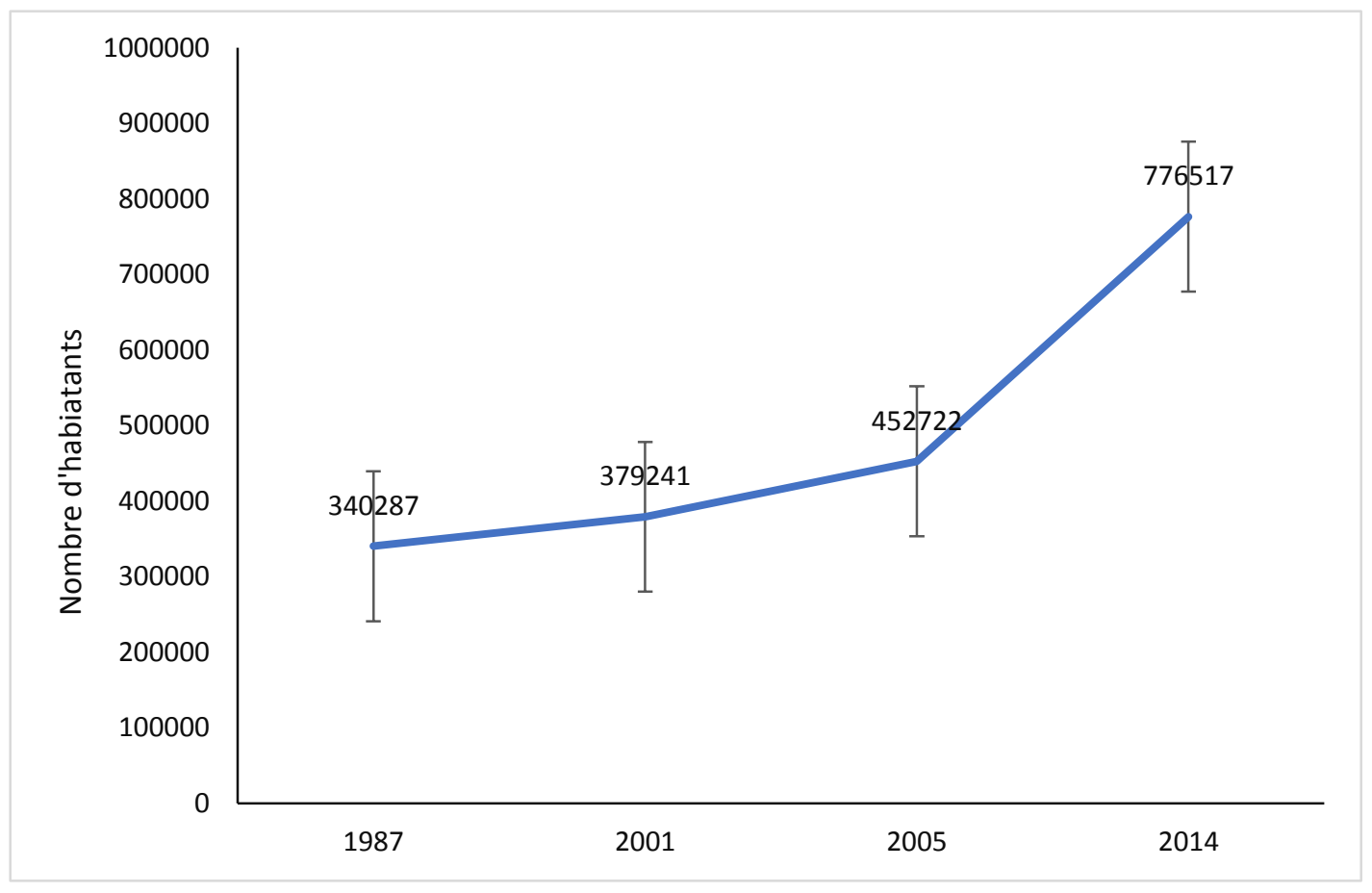

Figure 7 : Evolution de la population du Moungo entre 1987 et 2014.

\section{DISCUSSION}

Sur la base des résultats cartographiques, 07 unités d'occupation du sol ont été identifiées dans le Moungo. Ces unités ne sont pas toujours bien différenciées à cause probablement des réponses spectrales proches pour ces formations végétales naturelles et particulièrement ligneuses. Ces difficultés ont été signalées par plusieurs auteurs et même dans d'autres pays (Avakoudjo et al., 2014 ; Mamane et al., 2018). Ces difficultés, pourront être liées à la définition des parcelles homogènes lors de choix des sites d'entrainement. Toutefois, malgré ces difficultés, les résultats obtenus restent satisfaisants.

L'analyse des cartes d'occupation du sol de 1986, 2001 et 2018 a permis de mettre en évidence l'évolution progressive de certaines unités d'occupation du sol entre ces trois années.

L'évaluation de la dynamique spatiotemporelle de la végétation du Moungo a révélé la régression des forêts denses et claires (formations naturelles) au profit des formations anthropiques (plantations industrielles et des champs de cultures et zone habitée) de 0,52\% par an. Les causes de la dégradation du couvert végétal sont d'origine anthropique et peuvent aussi être d'origine climatique lorsque la zone écologique ne bénéficie pas de la pluviosité minimale devant permettre la reconstitution spontanée des formations végétales, ce qui est de plus en plus observé avec les changements climatiques (Adjonou et al., 2010). De même, la superficie des formations végétales régresse en faveur de celle des activités anthropiques notamment les champs, les jachères et les agglomérations. De plus, les activités agricoles constituent les causes majeures de la dégradation de la végétation (Nguimdo, 2017 ; Solefack et al., 2018 ; Temgoua et al., 2018b ; Tanougong, 2019). Toutefois, cette régression des formations végétales naturelles s'accompagne de la perte de la biodiversité et de la dégradation des terres.

La forte densité de la population de cette zone constitue également l'une des causes de dégradation de ces formations végétales naturelles. En effet, dans le Moungo, selon le 
Rapport d'activités annuelles de la section Départementale des Enquêtes et des Statistiques Agricoles du Moungo, en 2014, l'effectif de la population est passé de 340287 habitants en 1987 à 776517 habitants en 2014. De même, la densité de la population est passée de 91,4 habitants $/ \mathrm{km}^{2}$ en 1987 à 208,57 habitants $/ \mathrm{km}^{2}$ en 2014 sur la même superficie. Ce résultat montre qu'en 27 ans seulement, la densité de la population a presque triplé. Cette augmentation de la population entraîne l'extension des zones habitées et des formations anthropisées. Par conséquent, la pression des populations sur le couvert végétal entraîne la diminution du peuplement forestier au profit des espaces agricoles. Cette disparition des formations végétales est à l'origine de l'augmentation des gaz à effet de serre dans l'atmosphère, interrompant ainsi l'équilibre écologique (Djohy et al., 2016). Ce résultat se rapproche de celui obtenu par Zakariyao et al. (2013) qui ont montré que, la forte croissance démographique enregistrée dans le Centre-Togo en 2010 serait à l'origine de la réduction des terres, la régression de leur surface voire même leur disparition.

La modification de paramètres climatiques ces dernières années au Cameroun, notamment la baisse des précipitations et la hausse des températures, induit une diminution de la disponibilité des ressources en eau et par conséquent, influence la production agricole et les formations végétales (Temgoua et al., 2018b).

L'agriculture est le principal moteur de la déforestation. Les surfaces cultivées et les savanes herbeuses entre 1986 et 2018 ont augmenté et varient respectivement de 2 159,12 ha et 51566,41 ha en 32 ans et représentent $13,87 \%$ de la déforestation dans le Moungo. Une analyse profonde des causes de la déforestation au Cameroun indique que l'agriculture (avec des petites exploitations agricoles de moins d'un hectare) représente plus de $60 \%$ de la dégradation du couvert forestier. En effet, une étude menée par le Ministère de l'Environnement, de la Protection de la nature et du Développement durable (MINEPDED) en 2018, dans la zone forestière bimodale au Cameroun, montre qu'entre 2001 et 2015 , la déforestation due à l'exploitation agricole est d'environ 220000 ha. De même, les résultats similaires ont été observés dans plusieurs autres localités du Cameroun (Solefack et al., 2018 ; Temgoua,et al., 2018b).

L'exploitation des ressources naturelles peut également être une cause de ce recul des formations naturelles. En effet, l'exploitation forestière anarchique par certains exploitants et sociétés forestières, ainsi que l'utilisation locale de certaines espèces comme bois de chauffage, bois d'œuvre, bois de service (Millicia excelsa, Pterocarpus, Khaya ivorensis, Pycnanthus angolensis, Baillonella toxisperma, ...), et pour la fabrication du charbon de bois (Khaya, Guibourtia, ...) conduit à la diminution des formations naturelles dans le Moungo.

Par ailleurs, la présence dans la zone des sociétés agro-industrielles telles que CARBAP, PHP, CDC, etc. pourraient forcément être une cause de la diminution des formations naturelles dans le Moungo. En effet, l'implantation de ces sociétés a entrainé un déplacement massif des populations vers cette zone du Moungo (plus de 21708 travailleurs permanents et saisonniers en 2012 seulement à la $\mathrm{CDC}$ ) avec pour conséquence une augmentation des zones habitées, et des champs cultivés (Assoua, 2013). De même, les superficies de la PHP exploitées sont passées de 1797 ha en 2007 à 6065 ha en 2012 (Assoua, 2013).

\section{Conclusion}

L'étude de la dynamique spatiotemporelle de l'occupation du sol dans le Moungo a montré que les formations végétales naturelles (forêts denses et claires) ont subi une forte tendance régressive au profit des formations anthropiques. Les savanes herbeuses, les plantations et les champs de culture ont subi une progression totale de $13,87 \%$ entre 1986 et 2018 malgré la tendance régressive qu'on a observé entre 1986 et 2001 due à des multiples crises connues par cette localité. Les forêts denses et claires ont connu un taux de régression de $16,53 \%$ de leur superficie entre 1986 et 2018. Cette régression de ces formations végétales particulièrement 
critique est due à plusieurs facteurs dont le plus important est la croissance démographique, l'extension des surfaces cultivées et l'exploitation forestière. Cette destruction du couvert végétal entraîne la dégradation du sol et surtout la perte de la biodiversité. Cette étude pose la nécessité d'assoir des mécanismes de contrôle plus efficaces sur la gestion des ressources naturelles de la zone et contribue par ailleurs à une meilleure connaissance des prédictions sur les évolutions des ressources naturelles attendues de différents scénarios soiéconomiques dans le Moungo.

\section{CONFLIT D'INTERETS}

Les auteurs déclarent qu'il n'y a pas de conflit d'intérêts.

\section{CONTRIBUTIONS DES AUTEURS}

Ce travail a été mené en collaboration avec tous les auteurs. MRT a réalisé la collecte des données sur le terrain et a écrit le manuscrit. MT a supervisé les travaux de cette étude, MLA a initié et dirigé cette étude. ADT a téléchargé et traité les images. Tous les auteurs ont lu et approuvé la version finale du manuscrit.

\section{REFERENCES}

Abdourhamane H, Morou B, Mahamane A, Saadou M, Issaka A. 2012. Caractérisation de la dynamique spatiotemporelle de l'occupation des terres dans le complexe des forêts de classées de Dan kada Dodo-Dan Gado (région de Maradi, Niger). Journal des Sciences de l'Environnement., 1(1): 16-26.

Adjonou K, Djiwa O, Kombate Y, Kokutse AD, Kokou K. 2010. Etude de la dynamique spatiale et structure des forêts denses sèches reliques du Togo : implications pour une gestion durable des aires protégées. Int. J. Biol. Chem. Sci., 4(1): 168-183. http://ajol.info/index.php/ijbcs

ASRT. 2013. Recueil des séries d'informations statistiques sur les activités économiques, sociales, politiques et culturelles de la Région du Littoral jusqu'en 2014. P. 393.
Assoua EN. 2013. Plantations industrielles de bananiers au Cameroun: cas des plantations de la région du Sud-Ouest. Rapport CIRAD, P. 31.

Avakoudjo J, Mama A, Toko I, Kindomhou V, Sinsin B. 2014. Dynamique de l'occupation du sol dans le Parc National $\mathrm{du} \mathrm{W}$ et sa périphérie au nord-ouest du Benin. Int. J. Biol. Chem. Sci., 8(6): 26082625.

DOI: http://dx.doi.org/10.4314/ijbcs.v8i6.22

Ballo A, Traoré SS, Coulibaly B, Diakité CH, Diawara M, Traoré A. 2016. Pressions anthropiques et dynamique d'occupation des terres dans le terroir de Ziguéna, zone cotonnière du Mali. European Scientific Journal., 12(5): 90-99. DOI : http://dx.doi.org/10.19044/esj.2016. v12n5p90

Bozongo J. 2018. Les déterminants de la déforestation : cas du Bassin du Congo. Annales de l'Université Marien N'GOUABI., 18(2): 45-56.

Ciza SK, Milwa JF, Malekezi AC, Gond V, Bosela FB. 2015. Identification des moteurs de déforestation dans la région d'Isangi. République Démocratique du Congo. Bois et Forêts des Tropiques., 324(2): 29-38. DOI: http://dx.doi.org/10.19182/bft.324a.3126 4

Djohy GL, Vodounon TH, Kinzo EN. 2016. Dynamique de l'occupation du sol et évolution des terres agricoles dans la commune de Sinende au Nord-Benin. Cahier du CBRST, Lettres, Sciences Humaines et Sociales, (9): 101-121.

FAO. 2010. Global Forest Fire Assessment 2000-2010. Working Paper, Rome, P. 495.

FAO. 2014. Méthodologie proposée pour l'analyse des agents et causes de la déforestation dans les sites pilotes composante 4 du Projet FFEM. Working paper, Rome, P. 365.

Hussain M, Chen D, Cheng A, Wei H, Stanley D. 2013. Change detection from remotely sensed images : From pixel based to object-based approaches. ISPRS Journal of Photogrammetry and Remote Sensing., 
80:

$$
\text { 91-106. }
$$

DOI :

http://dx.doi.org/10.1016/j.isprsjprs.2013 .03 .006

Inoussa MM, Mahamane A, Mbow C, Saadou M, Yvonne B. 2011. Dynamique spatiotemporelle des forêts claires dansle parc national du W du Niger (Afrique de l'Ouest). Science et Changements Planétaires/Sécheresse., 22(2): 108-116. DOI :

http://dx.doi.org/10.1684/sec.2011.0305

Mamane B, Amadou G, Barrage M, Comby J, Ambouta JMK. 2018. Dynamique spatiotemporelle d'occupation du sol dans la Réserve Totale de Faune de Tamou dans un contexte de la variabilité climatique (Ouest du Niger). Int. J. Biol. Chem. Sci., 12(4): 1667-1687. DOI: http://dx.doi.org/10.4314/ijbcs.v12i4.13

Ndobe SN, Mantzel K. 2014. Déforestation, et REDD dans le Parc National de Takamanda au Cameroun. https://WWW.forestpeople.org, consulté le $6 / 09 / 2019$.

Nguimdo V. 2017. Dynamique de la déforestation, de la dégradation et des stocks de carbone dans la forêt d'Enseignement et de Recherche de l'Université de Dschang à Bélabo, EstCameroun. Mémoire d'Ingénieur de Conception des Eaux, Forêts et Chasses, Université de Dschang, P. 79.

Smith R.B. 2012. Introduction to remote sensing of the environment. Tutorial, P. 32.

Solefack MC, Njouonkou AL, Temgoua LF, Djouda R, Zangmene JB, Ntoupka M. 2018. Land-Use/ Land-Cover change and Anthropogenic Causes Around Koup Matapit Gallery Forest, West-Cameroun. Journal of Geography and Geology.,
10(2): 201-219. DOI: http://dx.doi.org/10.5539/jgg.v10n2p56

Tanougong NA. 2019. Modélisations prédictives des changements d'occupation des sols à l'horizon 2035 : cas du massif forestier intercommunal de Bélabo-Doumé-Diang, dans la Région de l'Est-Cameroun. Mémoire de Master of Science en foresterie, Université de Dschang, P. 137.

Temgoua LF, Ajonina G, Woyu HB. 2018a. Land Use and Land Cover Change Analysis in Ajei Upland Waterched Community Forest, North West Region, Cameroon. Journal of Geoscience and Environment Protection., 6(09): 83-99. DOI : https://doi.org/10.4236/gep.2018.69007

Temgoua LF, Solefack MC, Nguimdo V, Tagne BC, Tanougong A. 2018b. Spatial and temporal dynamic of landcover/land-use and carbon stocks in Eastern Cameroun : a case study of the teaching and research forest of the University of Dschang. Forest Science and Technology., 14(4): 181-191. DOI : https://doi.org/10.1080103.2018.152074 3

Zakariyao K, Abdourazakou A, Minkilabe D, Tchaa B, Ama-Edi K. 2013. Dynamique spatio-temporelle de l'occupation du sol dans le Centre-Togo. Revue de Géographie du Lardymes., 7(10) : 163172. DOI : https://hal.archivesouvertes.fr/hal-01564256 